\title{
Hard X-ray behaviour of Her X-1 during second main-on after the anomalous low state
}

\begin{abstract}
R. K. Manchanda ${ }^{\star}$
Tata Institute of Fundamental Research, Mumbai, India

Received 16 July 2002 / Accepted 13 February 2003

Abstract. The binary X-ray pulsar Her X-1 shows a variety of time variations both in luminosity and its pulse features. For the first time an about 600 days long anomalous low state was seen in the light curve of ASM on board RXTE during the period MJD 51200-51800. We observed the source in the 20-200 keV energy band during its second main-on in the post anomalous state on November 21, 2000. The source was found to be extremely bright at high energies and a total of 26000 photons from the source were detected during the $35 \mathrm{~min}$ snapshot observation. The cyclotron absorption line at $\sim 34 \mathrm{keV}$ and its second harmonics were clearly detected in the data. We present the spectral details and discuss its implication in terms of the geometrical properties of the source.
\end{abstract}

Key words. accretion, accretion disks - X-rays: stars - pulsars: individual - Her X-1

\section{Introduction}

The binary X-ray pulsar Her X-1 is a template system for the low mass X-ray binaries group. The source exhibits a variety of temporal variations like the $1.24^{\mathrm{s}}$ pulsation, $1.7^{\mathrm{d}}$ binary period, 35 day cyclic variation of high and low fluxes during which source remains on for about 5 binary cycles, Mid-on emission during the $35 \mathrm{~d}$ cycle, and the possible $1.6^{\mathrm{d}}$ periodicity in the intensity dips seen clearly from the source outside the eclipse phase (Tananbaum et al. 1972; Deeter et al. 1981).

The X-ray spectrum of Her X-1 in the low energy region below $30 \mathrm{keV}$ is characterized by a power law with index $\alpha \sim 1$ between 2-20 keV and a sharp cut-off beyond showing a power law fit with $\alpha \sim 4.5$ or a thermal temperature of $k T \sim 5-7 \mathrm{keV}$ (Mihara et al. 1991). However, in the broad band observations up to $100 \mathrm{keV}$, an additional power law component with $\alpha \sim 1.5$ is required to best fit the data (Manchanda et al. 1973, 1984; Soong et al. 1990). A prominent cyclotron absorption line feature between $35-55 \mathrm{keV}$ distinguishes its spectrum from the other known LMXB pulsating $\mathrm{X}$-ray sources. The high energy line feature is interpreted as arising due to cyclotron absorption in the intense magnetic field region $\left(\sim 10^{12} \mathrm{G}\right)$ near the magnetic poles of the rotating neutron star (Truemper et al. 1978). A second cyclotron absorption feature corresponding to the second harmonics has been observed on occasions (Manchanda 2001). However, there is no common consensus as yet on the line width and the centroid energy. The data suggest an apparent correlation with the binary phase, 35 day cyclic phase as well as the source luminosity (Staubert et al. 1983; Voges et al. 1982;

\footnotetext{
* e-mail: ravi@tifr.res.in
}

Tueller et al. 1984; Mihara et al. 1991, 1998; Gruber et al. 1998; Soong et al. 1990; Dal Fiume et al. 2000).

A binary system containing a $1.3 M_{\odot}$ neutron star with its optical companion HZ Her fits quite well the observations both in optical and in the X-ray band (Doxsey et al. 1973). Pulse features and the bulk spectral properties of the source can be explained by the mass accretion on to the neutron star from the accretion disk, which in turn is fed by the mass flow from the companion star. However, there is no universally accepted model for explaining the $35^{\mathrm{d}}$ on-off cycle of the source. A variety of mechanisms have been proposed in literature (Roberts 1974; Petterson 1975; Gerend \& Boynton 1976; Priedhorsky \& Holt 1987; van Kerkwijk et al. 1998). The poor quality of the 35 day clock leads to some of the difficulties in invoking precise mechanisms (Oegelman 1987). The origin of the fourth $.8^{\mathrm{d}} / 1.6^{\mathrm{d}}$ clock is still unknown (Crosa \& Boynton 1980; Truemper et al. 1986; White et al. 1995).

Since its discovery, a regular 35 day on-off cycle in the source intensity has been the key characteristic of the source, and hence its geometrical models. The source intensity remains high for $\sim 10$ days during the main-on state and for $\sim 5$ days during the mid-on state half way between the cyclic off state. The cycle regularity is clearly visible in the light curve of the source in the BATSE archival data since 1991 in the hard X-ray energy band of 20-100 keV and in the ASM data since 1994 in the soft X-ray band. A low intensity behaviour of Her X-1 at low energy was first observed in 1983 from the EXOSAT data during June to August (Parmar et al. 1985). No contemporary data is available in the hard X-ray band. However, the non detection of the source by ASCA in August 1993 in the soft X-ray band (Mihara \& Soong 1994) corresponded to only a reduced 
activity of the source in the hard X-ray band as is seen from the BATSE data.

The source entered an anomalous low intensity state in March 1999 which persisted for about 18 months. As seen in the BATSE and the RXTE light curves, this recent anomalous phase was extended over the entire X-ray energy band of 0.1 to $200 \mathrm{keV}$ or possibly beyond. The ASM and BATSE light curve show a gradual drop in the X-ray luminosity of the sources until the anomalous low state (ALS) where the normal turn-on did not occur. Optical spectra of the HZ Her taken during ALS showed prominent emission lines features of CIII/NIII and He II at comparable strength to the normal main-on observations (Margon et al. 1999). Since these emission line features are produced due to Bowen pumping caused by X-ray irradiation of the companion star, it firmly establishes that even though no X-ray flux was detected from the source, the X-ray photon production in the emitting regions of the source continued at the same level as in the normal main-on state. The spectral data taken before the source ingress to the ALS, show that only significant variation was observed in the $n_{\mathrm{H}}$ value which increased to $\sim 5 \times 10^{23} \mathrm{~cm}^{2}$, all other parameters remained unchanged within the statistical uncertainties. The pulse period near to the ALS was measured to be $1237.77485 \mathrm{msec}$. A comparison of pulse period obtained during the preceding normal state, indicated that the source had switched to a rapid spin-down mode compared to its normal spin-up behaviour. (Parmar et al. 1999; Levine \& Corbet 1999; Coburn et al. 2000). Using BeppoSAX observations during the first main-on after the anomalous low state, Oosterbroek et al. (2001) have reported the evidence that the rapid spin down of the source continued during the entire ALS period and the source pulse period changed to the value observed in $1984 / 5$. The measured pulse period of 1237.7697 corresponds to a $\frac{\dot{P}}{P} \sim 3 \times 10^{-6} \mathrm{yr}^{-1}$. The pulse profiles and the spectral behaviour of the source in the 0.1 to $30 \mathrm{keV}$ energy band was found to be consistent with the earlier observations during high state.

In this paper, we present the hard X-ray observations in the extended energy region of 20-200 keV made in the middle of second main-on phase as seen in ASM light curve after the anomalous low state. This observation was planned for the midon phase in the 35 day cycle based on the old ephemeris. The source was found to be quite bright at hard X-ray energies. The observed time averaged spectrum is hard with a clear indication of cyclotron features. We have also used the RXTE archival data form the PCA detectors taken on the same day to constrain the model parameters.

\section{Observations}

The X-ray observations were carried out using LASE payload at a ceiling altitude of $2.5 \mathrm{~g} \mathrm{~cm}^{-2}$ during a balloon flight conducted on November 21, 2000 from Hyderabad, India. The newly developed balloon-borne payload code named LASE consists of an X-ray telescope made of three identical modules of specially developed large area scintillation detectors having both passive and active shielding and fitted on a fully steerable alt-azimuth mount. One of the primary goal of the LASE experiment is to look for periodic and quasi-periodic components

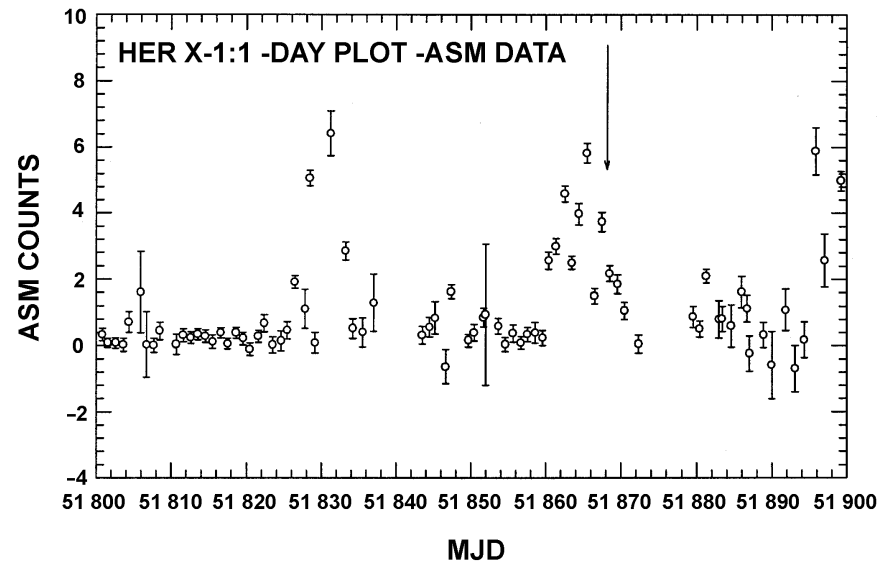

Fig. 1. X-ray light curve of Her X-1. The arrow in the figure represents the epoch of present observation.

in the hard X-ray region from LMXB and AGN sources in the hard X-ray energy region up to $200 \mathrm{keV}$ (Manchanda 1998).

Each detector module is a stand alone unit and consists of a combination of thin and thick sodium iodide detector arranged in a back-to-back configuration and has a geometric area of $400 \mathrm{~cm}^{2}$. The field of view of each module is $4.5^{\circ} \times 4.5^{\circ}$ and is defined by a slat collimator made from specially designed sandwiched material of Lead, Tin and Copper. The response matrix or each module is generated by using the preflight calibration data at different energies. Each module is also provided with a $\mathrm{Am}^{241}$ calibration source for gain calibration during the flight using ground command. The details of the detector design, associated electronics and the control system are given elsewhere (D'Silva et al. 1998). A $3 \sigma$ sensitivity of the LASE telescope in the entire energy range up to $200 \mathrm{keV}$ is $\sim 1 \times 10^{-6} \mathrm{~cm}^{-2} \mathrm{~s}^{-1} \mathrm{keV}^{-1}$ for a source observation of $10^{4} \mathrm{~s}$.

Her X-1 was observed for a continuous stretch of $35 \mathrm{~min}$ between 0630 and 0705 UT on November 21st and the source background was measured for $20 \mathrm{~min}$ each before and after the observation. The off-source pointing location was carefully selected blank field from the known X-ray source catalog. Present observations correspond to binary phase $\phi_{1.7}=0.04$ and $\phi_{35}=0.43$ (old ephemeris). Using the X-ray light curve from the all sky monitor on-board RXTE for the 35-day turnon corresponding to the present observations, the derived phase is about $\phi_{35} \sim 0.23$. The present observations correspond to MJD 51868.28.

\section{Results}

The present observations were timed to make a snap-shot spectral measurement of the source near the short mid-on in the 35 days cycle. In Fig. 1, we have plotted an expanded view of the X-ray light curve of the source taken from the all sky monitor on board RXTE. The arrow in the figure indicates the epoch of present observations. It is clearly seen from the figure that present data correspond to the main-on due to a delayed onset of the source as noted by Oosterbroek et al. (2001). It is also seen from the figure that in the post-anomalous state, the second main-on represents the normal behaviour during which 
the source intensity remained high for about 10 days, while the first main-on was of much shorter duration. A total excess of 26000 photons due to Her X-1 were recorded in the three detectors during the present observations. This corresponds to a combined statistical significance of $\sim 35 \sigma$.

In the LASE payload, a $25 \mu$ sec temporal resolution for each accepted event is achieved by referencing the arrival time with the telemetry frame start pulse. To determine the pulse period, we computed a table of arrival times for all photons in the entire energy range. After making the barycentric conversion we binned the data and searched for a coherent pulsations around $1237.7 \mathrm{msec}$ using XRONOS folding routine which maximizes the $\chi^{2}$ deviation of the phase-bins from the phase averaged value. In the present data the best fit period corresponds to $1237.788 \pm 0.01 \mathrm{msec}$ and is quite consistent with the value of $1237.7697 \mathrm{msec}$, observed during first mid-on in the post-anomalous state of the source. The present data supports the continued rapid spin-down of Her X-1 during the ALS as inferred from the BeppoSAX data (Oosterbroek et al. 2001).

To derive the time averaged source spectrum, the source contribution was binned in 20 energy channels between 19.5 to $200 \mathrm{keV}$ band. Each bin in the grouped data contained more than 600 source photons. The ASCII data files were then converted to FITS format and analyzed using XSPEC spectral analysis model fitting routine. The FITS files of the detector response matrix, atmospheric transmission and the detector resolution function constitute the other key input parameters during the spectral fitting. A broken power law as well as a composite function with thermal and non thermal terms represent the continuum data equally well. Therefore, the model fits were made using both the broken power law and the composite model ( $k T$, $\alpha$ ) for the source continuum along with the cyclotron absorption features. The data folded with best fit broken power law model is shown in Fig. 2. The cyclotron absorption features were fitted with the Mihara's cyclotron function as provided in the XSPEC analysis tools. The del-chi in figure indicates the quality of the fit to the data and the improvement in the chisquare value of the fit was $55 \%$ with the cyclotron absorption lines. The best fit values of various parameters for both models are summarized in Table 1. The data presented in Fig. 1, clearly demonstrates that present observations corresponded to the main-on phase rather than the near mid-on expected on the basis of mean 35 day ephemeris. From a detailed analysis of the on-state data since 1996, Oosterbroek (2001) have derived that main-on in the post anomalous state are delayed by 7 days i.e. $\sim 0.2$ in phase. We can therefore, safely assume that the delay in the on-set was caused by the anomalous low state of the source. However, such large delay is very difficult to account in any reasonable geometrical model of the source. The measured pulse period in our data is consistent with the BeppoSAX measurements of rapid spin down of the source during ALS. Detection of the source with high significance during our observations clearly suggests that Her X-1 had regained all of its characteristics by the second main-on. The spectral data plotted in Fig. 2 show not only that the source was quite bright at energies above $30 \mathrm{keV}$, but also that the cyclotron absorption features are clearly seen in the data.
Table 1. Best fit parameters for LASE data.

\begin{tabular}{lcc}
\hline \hline Model & bknpower & bremss+power \\
\hline continuum & & \\
index 1 & $3.85 \pm 2.4$ & $2.8 \pm 6.3 \mathrm{keV}$ \\
index 2 & $1.33 \pm 0.14$ & $1.33 \pm 0.2$ \\
cyclotron line & & \\
$E_{1}$ (abs) & $34.24 \pm 2.42$ & $34.82 \pm 3.33$ \\
$E_{w 1}$ & $4.7 \pm 2.3$ & $4.96 \pm 2.5$ \\
$E_{d 1}$ & $1.44 \pm 0.91$ & $1.29 \pm 0.79$ \\
second harmonics & & \\
$E_{2}=2 \times E_{1}$ & & \\
$E_{w 2}$ & $12.3 \pm 3.2$ & $13.1 \pm 3.6$ \\
$E_{d 2}$ & $0.1 \pm 0.11$ & $0.11 \pm 0.12$ \\
$\chi^{2}$ (d.o.f.) & & \\
\hline
\end{tabular}

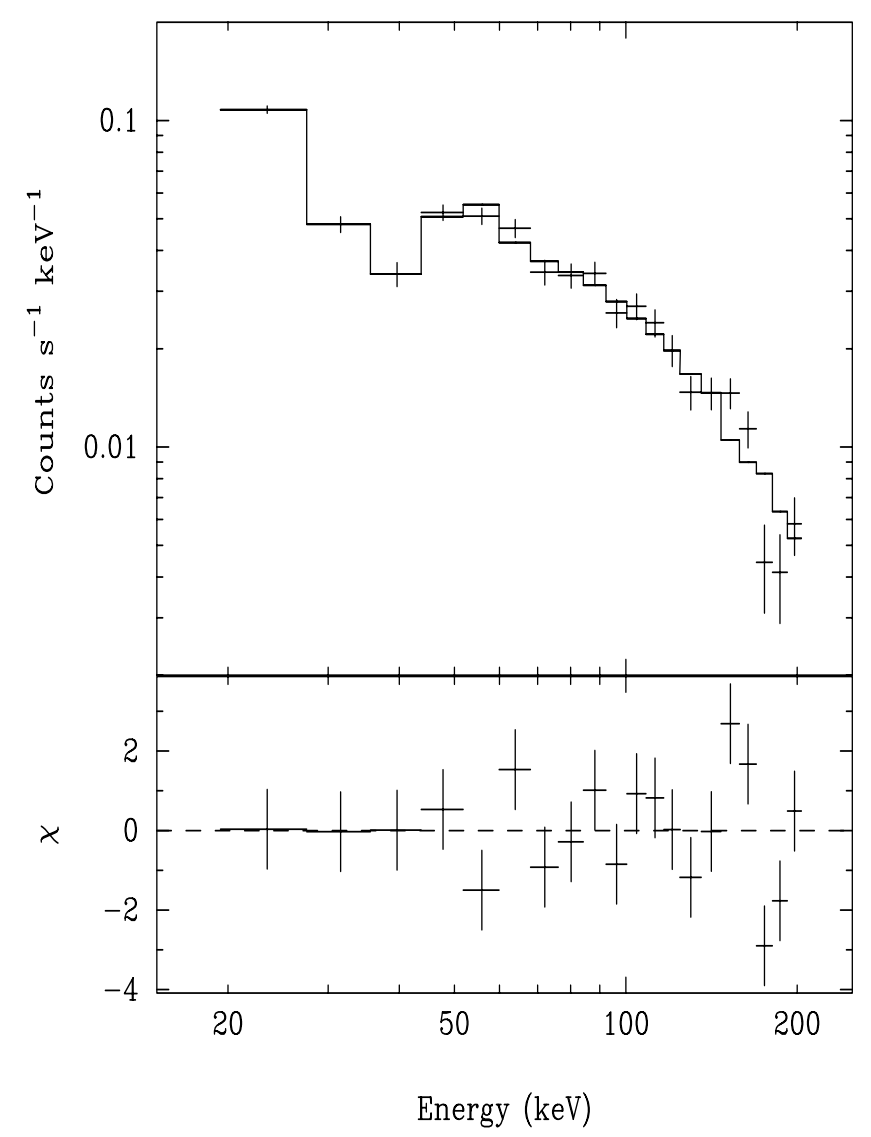

Fig. 2. Spectral fit for the LASE data. See Table 1 for details of various spectral components.

In order to further constrain the spectral parameters in the low energy region we have used archival data form PCA observation on board RXTE made on Nov. 21, 2000 at 0341-0553 UT. Fortunately, this observation was made only 4 hour before our observation in hard X-ray band and therefore, must represent a very high degree of correlation to the high energy data. Figure 3 shows the PCA and LASE data and the best fit value of various components. Since the PCA data above $40 \mathrm{keV}$ have large uncertainties even for bright 


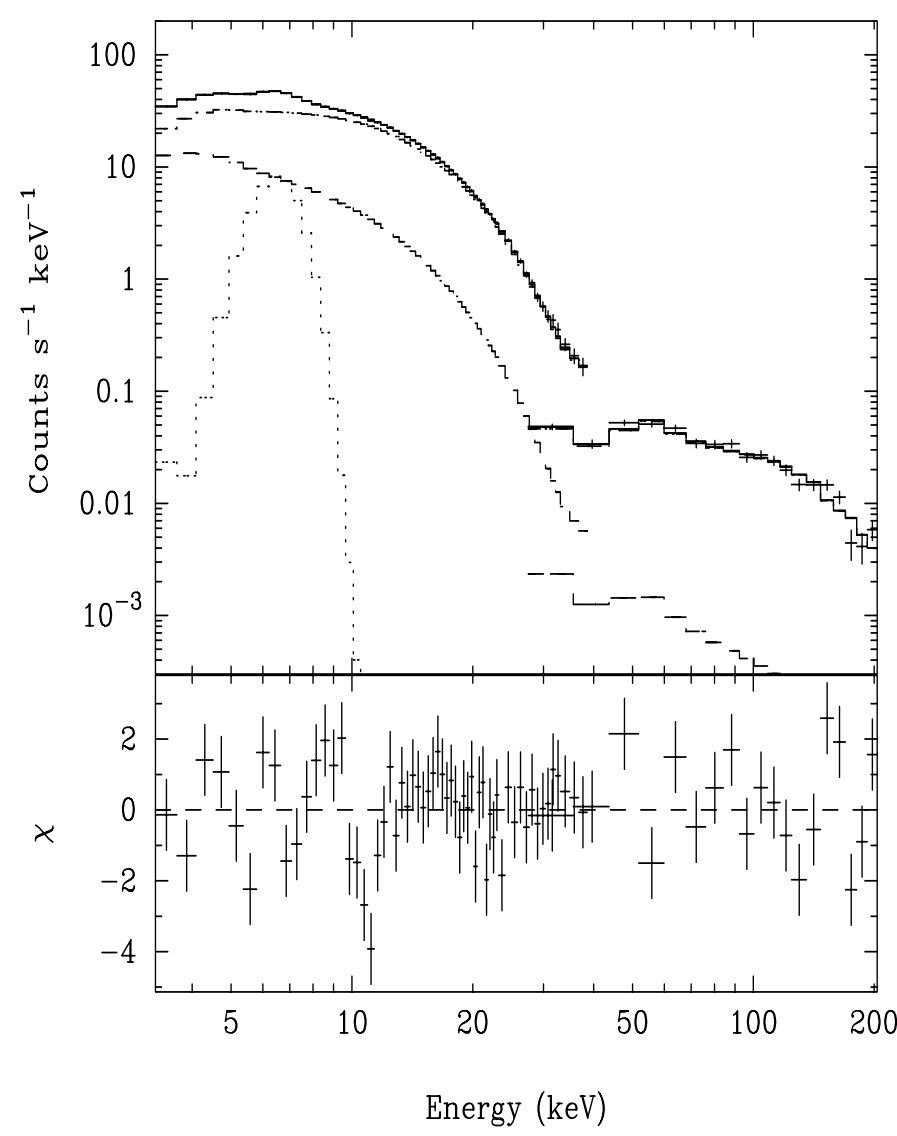

Fig. 3. Composite spectral fits to the LASE and PCA data.

sources, we have not utilized the data from higher energies. The model component used for the combined fit are a leaky warm absorber similar to the one proposed from the ASCA data (Endo et al. 2000) and consists of a Gaussian iron line, power law with a high energy cut-off, a second power law component and the cyclotron absorption line features as defined by the Mihara function. The values of different best-fit parameters are given in Table 2. The addition of the second harmonic improved the value chi-square from 3.98 to 1.68 per degree of freedom. The most important fact to note is that no arbitrary scaling or additive terms of any kind were necessary to make a combined fit to the data from the two instruments. This clearly points to the very well understood response matrix of the LASE payload. It is seen from the Table that best fit value of $n_{\mathrm{H}} \sim 5 \times 10^{22}$ derived for the combined fit is mid way between the zero value for the normal state and the $n_{\mathrm{H}} \sim 4 \times 10^{23}$ inferred for the low and anomalous low states (Coburn et al. 2001). To illustrate the hard X-ray behaviour in Fig. 4 we plotted a comparison of the present observations with the hard X-ray measurements made during 1997 by the same instrument. PDS data from BeppoSAX in the $2-30 \mathrm{keV}$ band corresponding to the first main-on is also plotted for comparison. It is clearly seen that not only the low energy data are consistent but the hard X-ray spectra above $50 \mathrm{keV}$ are also similar. The only main difference being the energy of the cyclotron absorption feature which occurred at $47.8 \pm 2 \mathrm{keV}$ in the 1997 data (Manchanda 2001).
Table 2. Best fit spectral components for PCA+LASE data.

\begin{tabular}{ll}
\hline \hline Warm absorber & $n_{\mathrm{H}} \sim 4.6 \pm 2.4 \times 10^{22}$ \\
Iron line & $6.57 \mathrm{keV}, \sigma \sim 0.56$ \\
Power law Index $\alpha_{1}$ & $0.94 \pm 0.36$ \\
$K_{1}$ & $7.4 \pm 2.8 \times 10^{-2}$ \\
Cut off & $16.6 \pm 1.1 \mathrm{keV}$ \\
Folding energy & $89.5 \pm 8.8 \mathrm{keV}$ \\
Power law Index $\alpha_{2}$ & $0.75 \pm 0.35$ \\
$K_{2}$ & $6.8 \pm 2.1 \times 10^{-2}$ \\
cyclotron line & \\
$E_{1}$ (abs) & $33.4 \pm 1.8$ \\
$E_{w 1}$ & $10.5 \pm 0.5$ \\
$E_{d 1}$ & $1.84 \pm 0.06$ \\
second harmonics & \\
$E_{2}=2 \times E_{1}$ & \\
$E_{w 2}$ & $30.2 \pm 4.0$ \\
$E_{d 2}$ & $0.62 \pm 0.06$ \\
$\chi^{2}$ (d.o.f.) & $1.68(64)$ \\
\hline
\end{tabular}

\section{Discussion}

In the standard picture of accretion in close X-ray binary systems, the all important transfer of mass from the primary to the compact companion takes place by Roche lobe overflow or by a stellar wind and secular changes in the spin period are associated with the magnitude of the accretion torque (Ghosh \& Lamb 1979). For the 35 day on-off cycle, the proposed geometries include, precession of a slave-disk, warp disk etc. (Roberts 1974; Petterson 1975; Pringle 1996; Shakura et al. 1999). A reduced accretion and a reduction in the inclination of the accretion disk has been proposed to be the cause for the ALS by Coburn et al. (2000). The reduced accretion leads to the decrease in the spin-up rate or even spin down in extreme circumstance, however, such a model does not account for the delay in the 35 day cycle which simply amounts to resetting the clock at an arbitrary epoch. It is to be noted that the effective change in the pulsar period consists of the sum of its normal spin up rate and the observed magnitude of the spin down rate. The rapid spin down as observed Her X-1, therefore, not only requires reduction in the on-going mass accretion but also an efficient pathway to loose angular momentum. The propeller effect is commonly assumed as the likely cause for reduced accretion (Illarionov \& Sunyaev 1975; Davies et al. 1979). However, this condition requires that the angular velocity of the star be larger than the Keplerian value of the angular frequency i.e. $\omega_{\mathrm{M}}>\omega_{\text {Kep }}$ such that spinning neutron star makes a centrifugal barrier and thereby mechanically expels the material. For such a condition to occur, one requires either a very fast spinning pulsar or extremely high magnetic field of $\sim 10^{15} \mathrm{G}$. On the other hand the heated wind model for mass loss requires higher temperature of the disk, which in turn implies a larger X-ray luminosity of the source (Illarionov \& Kompaneets 1990).

The observed X-ray and optical data clearly limits three vital parameters in the case of Her X-1. First, the magnetic field of $\sim 3 \times 10^{12} \mathrm{G}$ as inferred from the cyclotron absorption line, 


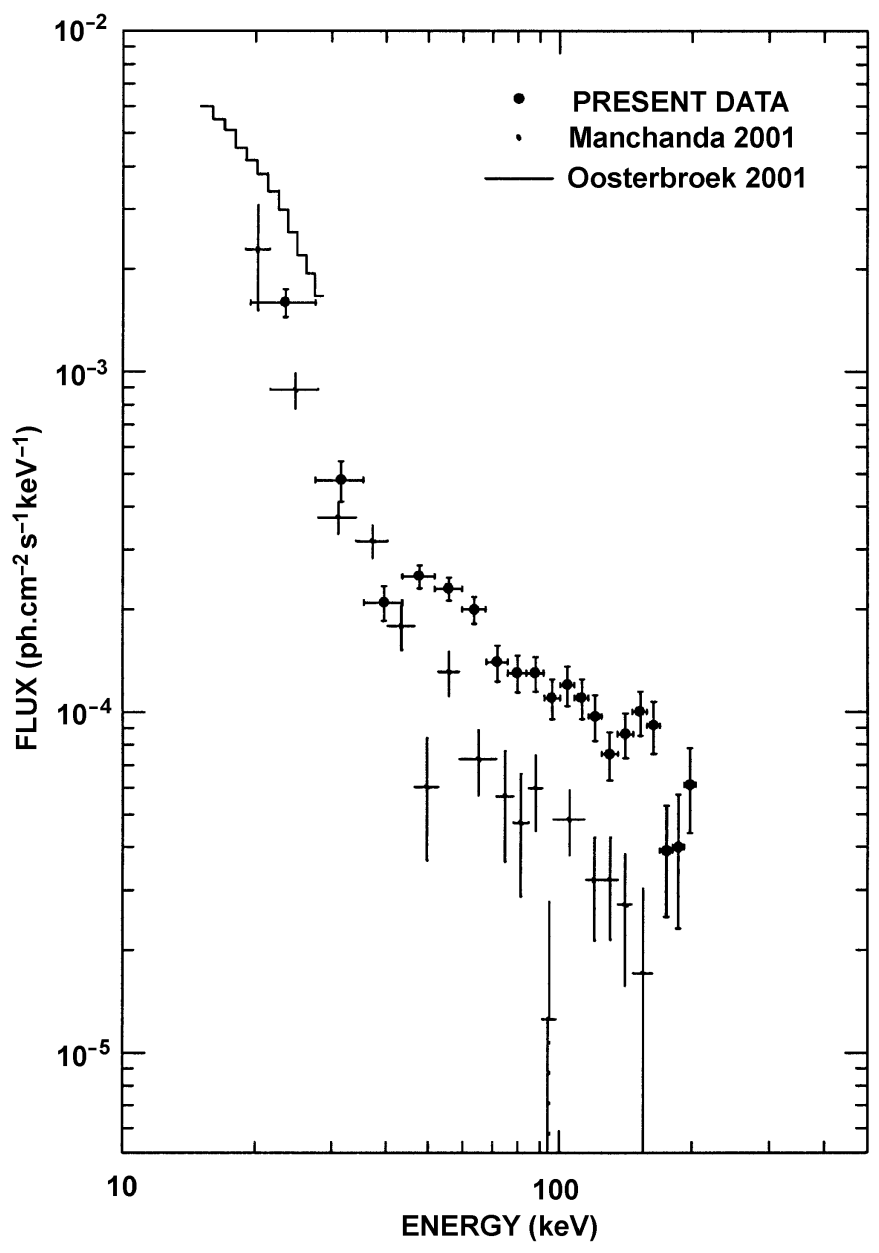

Fig. 4. Comparison of the pre and post ALS hard X-ray observations of Her X-1.

second, the X-ray production remained at the normal level during the ALS phase and third, the spin down of the source was rapid. A spin down model based on torque reversal due to warping of the accretion disk has been explored by van Kirkwijk et al. (1998). Apart from a large number of implausible assumptions necessary to explain the observed data, authors note that once the instability which leads to warping of the inner part of the disk is set, it is difficult to re-normalize the system. As mentioned earlier, about the large value of $n_{\mathrm{H}}$ observed during the ALS, it is tempting to relate the anomalous low state with an extended normal off-mode behaviour during the 35 day cycle. However, the observation of the phase difference of the main-on in the post ALS state as shown in the Fig. 5, based on the old ephemeris remains unresolved. In addition, though the non-detection of the X-ray flux from the source can be explained by obscuration in this model, the observation of rapid spin down of the source during the ALS would require an independent set of assumptions. In Fig. 5 we have plotted the long term light curve of the source in the pre and post ALS using ASM data. The dotted lines in the figure represent the expected on-set of the 35 day cycle. We have initialized the data visually at MJD 50807 corresponding to start of main-on. It is to be noted from the figure that unlike earlier observation of fluctuations on the mean ephemeris for 35 day period
(Oegelman 1987), the present phase shift appears to be of permanent nature to date.

Thus in the absence of any consistent geometrical model to explain both the spectral and temporal behaviour of the source, we propose that the emission of a strong jet from the Her X-1 led to the observed anomalous state of the source by causing an increased period of obscuration in the line of sight for about 18 months. A magneto-centrifugal wind has been discussed as the underlying mechanism for the spurting jets in which the ionized particles within the accretion disk are flung out along magnetic field lines that ultimately twist into a helical shape along the rotational axes of the disk. Orbital energy from the accretion disk is dissipated in propelling the polar jets (Königl 1989). Detailed models for the origin and self collimation of such jets from the surface of Keplerian accretion disks surrounding the central objects like YSO and blackholes have been discussed in literature (Pelletier \& Pudritz 1992). Similarly, the ejection of moderately relativistic jets powered by rotational energy has also been proposed based upon the observations of superluminal jets from micro-quasars (Zhang et al. 1997; King \& Kolb 1999). Such escaping jets not only lead to a large loss of gravitating mass but also efficiently extract specific angular momentum from the system i.e. the disk as well as the central object. Observationally, episodes of jet ejection have been observed for GRS 1915+105 (Mirabel \& Rodriguez 1994), SN 1987a (Manchanda et al. 1989) and Cyg X-3 (Mioduszewski et al. 1998). In addition, pre-flare quenching in hard X-ray band of $20-100 \mathrm{keV}$ has been noted for the large flare (Hjellming \& Han 1995). Therefore, an episode of jet ejection, will affect the geometry of the precessing accretion disk and also force a rapid spin down in the source. The escaping material will disrupt the inner accretion disk structure, which is dynamically stabilized by the precessing and the motion of the accreted material, thus leading to not only obscuration of the source but also it will cause a shift in the 35 day ephemeris, since the amplitude of the perturbation will die down at an arbitrary time scale independent of the established temporal behaviour of the source. It is seen from Fig. 5 that the phase shift of about 7 days seen during the first main-on (Oosterbroek et al. 2001) is a persistent feature of the source and is consistent with the conclusion of re-establishing the stability of the system randomly.

In the present model we need to address the question, as to what triggers such transient spurting of the jet. The steady state angular momentum equation of an axisymmetric accretion disk threaded by the magnetic field and undergoing viscous stress depends upon the angular momentum of the accreting matter $\rho u_{\mathrm{p}} \Omega r^{2}$, the ordered magnetic energy density in the disk $\frac{r B_{\phi} B_{\mathrm{p}}}{4 \pi}$ and the viscous torque $\tau_{\text {visc }}$. For a dipole field, the $B_{\phi}$ is taken as $B_{z}$. We propose that poloidal magnetic field in the accretion disk grows on arbitrary time scale depending upon the accretion rate and the development of instabilities in the disk. When sufficiently strong, this component can then drive the ejection of a jet. It has been shown that poloidal magnetic field may be dragged radially inward by the accreting gas until its dissipative escape, driven by the gradient in magnetic pressure, limits its growth. Provided that the magnetic 


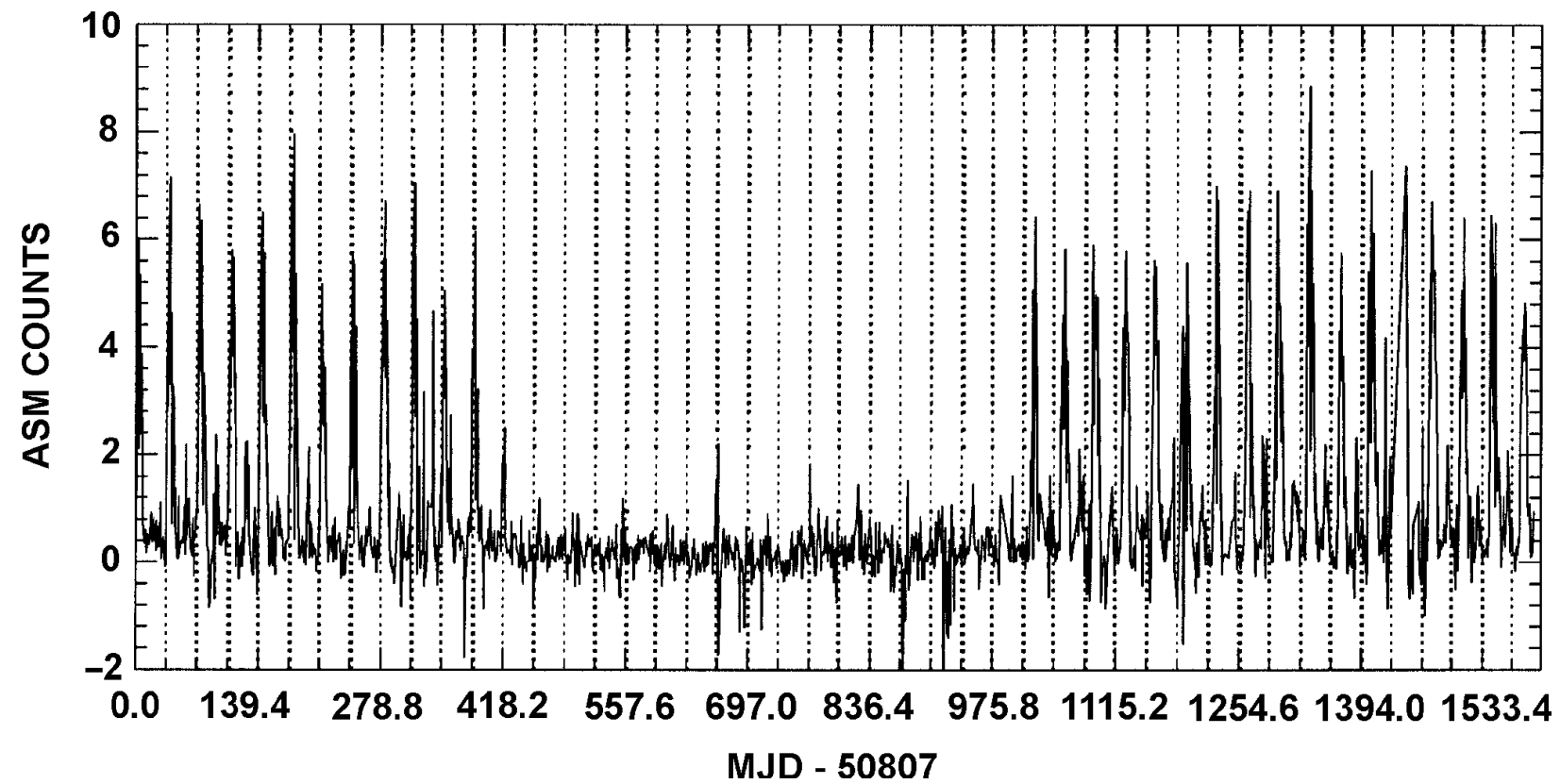

Fig. 5. Long term light curve of Her X-1. The dotted line represent the expected turn-on dates based on the old ephemeris.

field makes an angle of less than $60^{\circ}$ with the radius vector at the disk in Keplerian motion, it will be energetically favorable for the gas to leave the disk in a centrifugally driven wind (Blandford 1989; Königl \& Ruden 1992).

A large inclination of the magnetic axis with respect to the spin axis in the case of Her X-1 further helps the obscuration scenario for the anomalous low state, set off by the disruption of the precessing disk due to the escape of the material in the form of a jet. The high inclination of the magnetic axis with respect to the spin axis in Her X-1 can be inferred from the following. The low energy pulse profile of Her X-1 shows a double peaked main pulse and a extremely weak inter-pulse but at higher energies it is a the sharp peaked pulse profile. This clearly suggests that orientation of the spin axis is such that pulsed X-rays are emitted from the funnel of one of the magnetic pole having an hollow cone structure. Since the duty cycle of the pulse is approximately given by $\sim \Delta \alpha \times 2 \pi \sin \chi$, where $\Delta \alpha$ and $\chi$ are the beaming angle and the inclination of the magnetic pole to the rotation axis, a large duty cycle of the pulse clearly require large angle of inclination. In fact even in the normal main-on mode, the possibility that the X-ray emission cone almost grazes the disk has been inferred from the possible scattering of the beam necessary to account for the slight asymmetry in the pulse profile as well as explaining the $6.7 \mathrm{keV}$ iron-line seen in the Her X-1 spectrum (Bai 1980; Mihara et al. 1991). Therefore, a perturbation of the precessing disk can easily lead to the observed flux anomaly from the source. The present model can also account for the fluctuations in the mean ephemeris for the 35 day period observed during the pre ALS data, if we assume that a low intensity variable magneto-centrifugal wind from the accretion disk is a normal feature of the source at all times.

Acknowledgements. It is pleasure to thank sarvashri J. A. R. D'Silva, P. P. Madhwani, N. V. Bhagat, B. G. Baggage and Ms. N. Kamble for mechanical and electronic fabrication of the payload. I also thank
Mr. V. Rana and Dr. B. Paul for their help in reducing the RXTE data. RXTE teams are gratefully acknowledged for the archival data.

\section{References}

Bai, T. 1980, ApJ, 239, 99

Blandford, R. D. 1989, in Theory of Accretion Disks, ed. P. Meyer, W. Duschl, J. Frank, \& E. Meyer-Hofmeister (Dordrecht: Kluwer), 35 Coburn, W., Heindl, W. A., \& Wilms, J., et al. 2000, ApJ, 543, 351

Crosa, L., \& Boynton, P. E. 1980, ApJ, 235, 999

Dal Fiume, D., Orlandini, M., Cusumano, G., et al. 1998, A\&A, 329, L41

Davies, R. E., Fabian, A. C., \& Pringle, J. E. 1979, MNRAS, 186, 779 Deeter, J. E., Boynton, P. E., \& Pravado, S. H. 1981, ApJ, 247, 1003

Doxsey, R., Bradt, H. V., Levine, A., et al. 1973, ApJ, 182, L25

D'Silva, J. A. R., Madhwani, P. P., Tembhurne, N., \& Manchanda, R. K. 1998, NIM, A412, 342

Endo, T., Nagase, F., \& Mihara, T. 2000, PASJ, 52, 223

Gerend, D., \& Boynton, P. E. 1976, ApJ, 209, 562

Ghosh, P., \& Lamb, F. K. 1979, ApJ, 234, 296

Gruber, D. E., Heindl, W. A., Rothshield, R. E., et al. 1998, Nucl. Phys. B Proc. Suppl., 69, 174

Hjellming, R. M., \& Han, M. 1995, in X-ray binaries (Cambridge Univ. Press), 308

Illarionov, A. F., \& Kompaneets, D. A. 1990, MNRAS, 247, 219

Illarionov, A. F., \& Sunyaev, R. A. 1975, A\&A, 39, 195

King, A. R., \& Kolb, U. 1999, MNRAS, 305, 654

Königl, A. 1989, ApJ, 342, 208

Königl, A., \& Ruden, S. P. 1992, in Protostars and Planets III, ed. E. H. Levy, \& J. I. Lunine (Tucson: University of Arizona Press)

Levine, A. M., \& Corbet, R. 1999, IAU Circ., 7139, 2

Manchanda, R. K., Iyengar, V. S., Durgaprasad, N., et al. 1973, Nature, 244, 59

Manchanda, R. K., Ubertini, P. U., Polcaro, V. F., et al. 1984, Astrophys. Lett., 24, 27

Manchanda, R. K., Sood, R. K., \& Waldron, L. 1989, A\&A, 211, 353

Manchanda, R. K. 1998, Adv. Space Res., 21, 1019 
Manchanda, R. K. 2001, BASI, 29, 25

Margon, B., Deutsch, E. W., Leinhardt, M., \& Anderson, S. F. 1999, IAU Circ., 7144

Mihara, T., Makishima, K., Ohashi, T., Sakao, T., \& Tashiro, M. 1990, Nature, 346, 250

Mihara, T., Ohashi, T., Makishima, K., et al. 1991, PASJ, 43, 501

Mihara, T., \& Soong, Y. 1994, New horizons of X-ray astronomy, ed. F. Makino, \& T. Ohashi (Universal Academy Press), 419

Mihara, T., Makishima, K., \& Nagase, F. 1998, Adv. Space Res., 22, 987

Mirabel, I. F., \& Rodriguez, L. F. 1994, Nature, 375, 464

Mioduszewski, A. J., Hjellming, R. M., Rupen, M. P., et al. 1998, ASP Conf., 144, ed. J. A. Zeusus, G. B. Taylor, \& J. M. Wrobel

Oegelman, H. 1987, A\&A, 172, 79

Oosterbroek, T., Parmar, A. N., Orlandini, M., et al. 2001, A\&A, 375 , 922

Parmar, A. N., Pietsch, W., McKechnie, S., et al. 1985, Nature, 313, 119

Parmar, A. N., Oosterbroek, T., dal Fiume, D., et al. 1999, A\&A, 350, L5

Petterson, J. A. 1975, ApJ, 201, L61

Pelletier, G., \& Pudritz, R. E. 1992, ApJ, 394, 117
Priedhorsky, W. C., \& Holt, S. S. 1987, Space Sci. Rev. E, 45, 291

Pringle, J. E. 1996, MNRAS, 281, 357

Roberts, W. J. 1974, ApJ, 187, 575

Shakura, N. I., Prokhorov, M. E., Postnov, K. A., \& Ketsaris, N. A. 1999, A\&A, 348, 917

Soong, Y., Gruber, D. E., Peterson, L. E., \& Rothschild, R. E. 1990, ApJ, 348, 641

Staubert, R., Bezler, M., \& Kendiziorra, E. 1983, A\&A, 117, 215

Tananbaum, H., Gursky, H., Kellog, E. M., et al. 1972, ApJ, 174, L14

Truemper, J, Pietsch, W., Reppin, C., et al. 1978, ApJ, 219, L105

Truemper, J., Kahabaka, P., Oegelman, H., Pietsch, W., \& Voges, W. 1986, in evolution of Galactic X-ray Binaries, ed. J. Truemper, W. H. G. Lewin, \& W. Brinckmann (Reidel), 239

van Kerkwijk, M. H., Chakrabarty, D., Pringle, J. E., \& Wijers, R. A. M. J. 1998, ApJ, 499, L27

Tueller, J., Cline, T. L., Teegarden, B. J., et al. 1984, ApJ, 279, 177

Voges, W., Pietsch, W., Reppin, C., et al. 1982, ApJ, 263, 803

White, N., Nagase, F., \& Parmar, N. 1995, in X-ray binaries, ed. W. H. G. Lewin, J. van Paradijs, \& E. P. J. van den Heuvel (Cambridge), 1

Zhang, S. N., Cui, W., \& Chen, W. 1997, ApJ, 482, L155 